\title{
Effect of Er doping on the superconducting properties of porous $\mathrm{MgB}_{2}$
}

\author{
O ERDEM ${ }^{1,2, *}$ and E YANMAZ ${ }^{2}$ \\ ${ }^{1}$ Faculty of Education, Department of Primary Education, Bayburt University, 69000 Bayburt, Turkey \\ ${ }^{2}$ Faculty of Sciences, Department of Physics, Karadeniz Technical University, 61080 Trabzon, Turkey
}

MS received 21 February 2014; revised 03 May 2014

\begin{abstract}
MgB}_{2}$ bulk sample with porous structure was produced by using the in-situ solid-state reaction method under argon (Ar) atmosphere of 10 bar. Elemental $\mathrm{Er}$ in powder form was doped into $\mathrm{MgB}_{2}$ with different compositions $\left(\mathrm{Mg}_{1-x} \mathrm{Er}_{x}\right) \mathrm{B}_{2}$, where $x=0.00,0.03$ and 0.05 , in order to investigate the effect of rare-earth (RE) element $\mathrm{Er}$ on the structural and electromagnetic properties of porous $\mathrm{MgB}_{2}$. The Er-doped samples result in small grain size structure compared to the undoped one. The lattice constants $a$ and $c$ of the doped samples, determined from $\mathrm{X}$-ray diffraction (XRD) analysis, increase with the increasing Er content, and consequently the superconducting transition temperature ( $T_{\mathrm{c}}^{\text {onset }}$ ) of $\mathrm{MgB}_{2}$, determined from resistivity measurements, is slightly suppressed. Also, the upper critical field $\left(B_{\mathrm{c} 2}\right)$, the irreversibility field $\left(B_{\text {irr }}\right)$ and the critical current density $\left(J_{c}\right)$ values are significantly enhanced in the doped samples. For the best sample $(x=0.03)$, at $15 \mathrm{~K}$ under a magnetic field of $4 \mathrm{~T}$, the $J_{\mathrm{c}}$ value reaches $2.4 \times 10^{4} \mathrm{~A} \mathrm{~cm}^{-2}$, which is higher than that of the porous sample by an order of $10^{3}$, and the $B_{\text {irr }}$ value at $20 \mathrm{~K}$ reaches $9.7 \mathrm{~T}$. These results imply that the RE element Er fills the pores, enhances the density and the grain connectivity. Hence, the superconducting properties of the porous $\mathrm{MgB}_{2}$ sample improve by $\mathrm{Er}$ doping.
\end{abstract}

Keywords. Superconductivity; $\left(\operatorname{Mg}_{1-x} \operatorname{Er}_{x}\right) B_{2}$; upper critical field; irreversibility field; critical current density.

\section{Introduction}

The discovery of superconductivity in $\mathrm{MgB}_{2}{ }^{1}$ has stimulated great scientific and technological interest, not only because of its simple electronic structure and high critical temperature $\left(T_{\mathrm{c}}\right),{ }^{1}$ but also due to its high critical current density $\left(J_{\mathrm{c}}\right){ }^{2}$ large coherence length ${ }^{3}$ and the absence of weak-link effect. ${ }^{4}$

It is well known that the rapid drop in $J_{\mathrm{c}}$ with the increasing applied magnetic field is one of the major problems for $\mathrm{MgB}_{2}$-based superconductor technologies. A rapid drop in $J_{\mathrm{c}}$ indicates low irreversibility field $\left(B_{\text {irr }}\right)$ and poor pinning ability due to a lack of effective pinning centres in $\mathrm{MgB}_{2}$. The density also has an important effect on the superconducting performance of $\mathrm{MgB}_{2}$. It is well known that the high occupation fraction of pores in sintered $\mathrm{MgB}_{2}$ bulk samples decreases the density and the effective cross-section area for carrying current. ${ }^{5}$ If the porosity can be decreased and the density can be increased, the critical current density of $\mathrm{MgB}_{2}$ will be increased significantly. It is well known that the resistivity of dense $\mathrm{MgB}_{2}$ is smaller than that of porous one. ${ }^{6}$ Also, the $J_{\mathrm{c}}(H=0)$ of $\mathrm{MgB}_{2}$ is inversely proportional to $\Delta \rho(\Delta \rho=\rho(300 \mathrm{~K})-\rho(50 \mathrm{~K}))$. This follows because the ratio of $\Delta \rho$ in fully dense and clean $\mathrm{MgB}_{2}$ to $\Delta \rho$ in $\mathrm{MgB}_{2}$ sample is a measure of the reduction in current-carrying area. $^{7}$

It is possible to improve the pinning behaviour of $\mathrm{MgB}_{2}$ by many methods, such as adding or doping ${ }^{8,9}$ and the

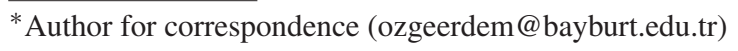

introduction of defects by irradiation, ${ }^{10}$ etc. Chemical doping is known to be one of the most effective methods in improving the superconducting properties of $\mathrm{MgB}_{2}$. Doping can substitute dopant atoms for atoms in $\mathrm{MgB}_{2}$ crystal lattice to increase the upper critical field $\left(B_{\mathrm{c} 2}\right)$ and induce defects and precipitates to increase flux pinning centres of $\mathrm{MgB}_{2} .{ }^{9}, 11$ However, the results of chemical doping are categorized into two types, as reported by Zhao et al: ${ }^{12}$ the doping which leads to the decrease of $T_{\mathrm{c}}$ and the loss of superconductivity, such as doping of $\mathrm{Al},{ }^{13}$ and the other doping which cannot dope the element in the $\mathrm{MgB}_{2}$ crystal lattice and has no effect on $T_{\mathrm{c}}$, such as Be doping. ${ }^{14}$ The second type of doping creates non-superconducting phases, which may work as pinning centres if a proper microstructure of the second phases can be formed in $\mathrm{MgB}_{2}$. It has been reported by Feng et al ${ }^{15}$ and $\mathrm{Zhao} \mathrm{et} \mathrm{al}^{12}$ that doping of $\mathrm{MgB}_{2}$ with $\mathrm{Zr}$ and Ti, respectively, increases the sample density, reduces the porosity and increases the $J_{\mathrm{c}}$ values.

Magnetic impurities usually have a stronger interaction with magnetic flux lines than non-magnetic impurities. If the impurities can be properly introduced into the $\mathrm{MgB}_{2}$ matrix, they may exert a stronger force to trap the flux lines and improve the pinning behaviour of $\mathrm{MgB}_{2}$. The magnetic doping elements, such as $\mathrm{Mn}, \mathrm{Fe}, \mathrm{Co}$ and $\mathrm{Ni}$, which can depair Cooper pairs, often degrade the superconducting performance of $\mathrm{MgB}_{2}$ in a magnetic field due to the presence of a local magnetic field. ${ }^{16-18}$ On the other hand, rare-earth (RE) elements often possess a strong magnetic moment, however, they do not suppress the superconductivity of $\mathrm{MgB}_{2} \cdot{ }^{19-21}$ When RE oxides and elements are doped into $\mathrm{MgB}_{2}$, they 
form impurity phases with boron, which can act as effective pinning centres in $\mathrm{MgB}_{2}$. The main resultants are $\mathrm{REB}_{6}$ for light REs ( $\mathrm{La}, \mathrm{Ce}, \mathrm{Pr}, \mathrm{Nd}, \mathrm{Sm}, \mathrm{Eu}, \mathrm{Gd}$ ) and $\mathrm{REB}_{4}$ for heavy RE elements (Tb, Dy, Ho, Er). Also the density of the doped samples increases compared with the undoped one. ${ }^{22}$ Doping with RE oxides $\operatorname{Pr}_{6} \mathrm{O}_{11}$ and $\mathrm{Eu}_{2} \mathrm{O}_{3}$ provides strong flux pinning due to the $\mathrm{PrB}_{6}$ and $\mathrm{EuB}_{6}$ impurity phases and increases $J_{\mathrm{c}}(H), H_{\mathrm{c} 2}$ and $H_{\text {irr }}$ of $\mathrm{MgB}_{2} . J_{\mathrm{c}}$ values at $10 \mathrm{~K}$ under a field of $5 \mathrm{~T}$ for undoped $(x=0.00), \operatorname{Pr}_{6} \mathrm{O}_{11}$-doped $(x=0.03)$ and $\mathrm{Eu}_{2} \mathrm{O}_{3}$-doped $(x=0.05) \mathrm{MgB}_{2}$ samples have been found to be $2.21 \times 10^{3}, 1.15 \times 10^{4}$ and $1.10 \times$ $10^{4} \mathrm{~A} \mathrm{~cm}^{-2}$, respectively. ${ }^{23}$ In total $0.1-10 \% \mathrm{Ho}_{2} \mathrm{O}_{3}$-doped $\mathrm{MgB}_{2}$ samples show no change in its crystal structure, $T_{\mathrm{c}}$ and $H_{\mathrm{c} 2}$, but exhibit significant enhancement in $J_{\mathrm{c}}$ and $H_{\mathrm{irr}}{ }^{24} \mathrm{~A}$ similar study of RE oxide-doped $\mathrm{MgB}_{2}$ samples, where RE oxides $=\mathrm{La}_{2} \mathrm{O}_{3}, \mathrm{Nd}_{2} \mathrm{O}_{3}, \mathrm{~Tb}_{4} \mathrm{O}_{7}, \mathrm{Er}_{2} \mathrm{O}_{3}, \mathrm{Lu}_{2} \mathrm{O}_{3}$, has shown that the doping causes no change in $T_{\mathrm{c}}$ and does not lead to a serious decrease in $J_{\mathrm{c}}$, despite the generation of larger amounts of impurity phases, such as RE borides and $\mathrm{MgO} .{ }^{22}$

In the literature, there are very few studies on the doping effects of RE elements on the superconductivity of $\mathrm{MgB}_{2}$. Therefore, more investigation needs to be done on doping $\mathrm{MgB}_{2}$ with RE elements to find possible options for improving the superconducting performance of this compound. In this work, the doping effects of Er on the superconducting properties of porous $\mathrm{MgB}_{2}$ have been studied.

\section{Experimental}

$\left(\mathrm{Mg}_{1-x} \mathrm{Er}_{x}\right) \mathrm{B}_{2}$ samples with different stoichiometric ratios were prepared by the in-situ solid-state-reaction method. Commercially available high-purity powders of crystalline $\mathrm{Mg}$ (99.8\%), Er (99.9\%) and amorphous B (95-97\%) were weighed to give a nominal composition of $\left(\mathrm{Mg}_{1-x} \mathrm{Er}_{x}\right) \mathrm{B}_{2}$, where $x=0.00,0.03$ and 0.05 . The mixtures of $\mathrm{Mg}, \mathrm{B}$ and Er powders were thoroughly ground for $1 \mathrm{~h}$ in an agate mortar by hand in air. The mixtures, weighing about $2 \mathrm{~g}$, were pressed into rectangular pellets $10 \times 15 \mathrm{~mm}^{2}$ in size under $1.3 \mathrm{GPa}$ pressure. The pellets were put in a chrome tube and sintered at $650^{\circ} \mathrm{C}$ for $2 \mathrm{~h}$ under constant 10 bar Ar gas pressure. After the heat treatment, the chrome tube was cooled to room temperature by furnace cooling. Then, the second grinding process was performed. Finally, the obtained powders were pressed into the same rectangular pellet form under 1.3 $\mathrm{GPa}$ pressure and sintered at $750^{\circ} \mathrm{C}$ for $2 \mathrm{~h}$ under 10 bar Ar gas pressure, followed by furnace cooling to room temperature.

The phase compositions of the samples were determined from X-ray diffraction (XRD) patterns using a Rikagu D/Max-IIIC diffractometer with $\mathrm{CuK} \alpha$ radiation, and the microstructures of the samples were analysed by a scanning electron microscope (SEM, JEOL JSM 6400). The upper critical field $B_{\mathrm{c} 2}$ and the irreversibility field $B_{\text {irr }}$ were defined as $B_{\mathrm{c} 2}=0.9 R\left(T_{\mathrm{c}}\right)$ and $B_{\text {irr }}=0.1 R\left(T_{\mathrm{c}}\right)^{25}$ from the resistance $(R) v s$. temperature $(T)$ curves $(5 \mathrm{~mA} \mathrm{ac})$ obtained by a four-probe resistivity measurements. Also, the $T_{\mathrm{c}}$ values were obtained from the resistivity curves of the samples. The magnetic properties ( $M-H$ loops) of the samples were determined using a vibrating sample magnetometre within the Quantum Design Physical Properties Measurement System for the temperature ranges from 4.2 to $35 \mathrm{~K}$. The magnetic $J_{\mathrm{c}}\left(\right.$ in $\mathrm{A} \mathrm{cm}^{-2}$ ) of the samples was calculated from the $M(H)$ curves via $J_{\mathrm{c}}=20 \Delta M /[a V(1-a / 3 b)]$ on the basis of Bean's critical state model. ${ }^{26}$ In this relationship, $\Delta M$ (in emu) is the width of the $M(H)$ hysteresis. $V$ (in $\mathrm{cm}^{3}$ ), $a$ (in $\mathrm{cm})$ and $b$ (in $\mathrm{cm}$ ) are the volume, length and width of the sample $(a<b)$, respectively.

\section{Results and discussion}

Figure 1 shows the XRD patterns of the $\left(\mathrm{Mg}_{1-x} \mathrm{Er}_{x}\right) \mathrm{B}_{2}(x=$ $0.00,0.03$ and 0.05 ) samples. It is very apparent from figure 1 that the undoped sample has the major phase of $\mathrm{MgB}_{2}$, with a little amount of $\mathrm{MgO}$. Also, it can be seen that the boride impurities $\mathrm{ErB}_{4}, \mathrm{ErB}_{2}$ and also an oxide phase $\mathrm{Er}_{2} \mathrm{O}_{3}$ have formed. As clearly seen from figure 1, the main peak belonging to $\mathrm{MgB}_{2}$ at around $42.4^{\circ}$ has decreased in intensity and has broadened for the doped samples. It is an indication of reducing the crystallite size for the doped samples. Table 1 also shows the variation of the lattice parameters of all samples. The lattice parameters $a$ and $c$ of the porous undoped sample are smaller than that of the dense $\mathrm{MgB}_{2}$ reported in literature. The difference is possibly due to the porous structure of the undoped sample. It is well known that the lattice parameters of $\mathrm{MgB}_{2}$ decrease with the increasing porosity. ${ }^{27}$ Both the lattice parameters $a$ and $c$ increase with the increasing Er concentration, as given in table 1. It is expected from these results that some Er atoms have partially substituted into $\mathrm{MgB}_{2}$, possibly at $\mathrm{Mg}$ sites.

The SEM images show the porous structure (the black area) and the agglomeration of particles in the undoped

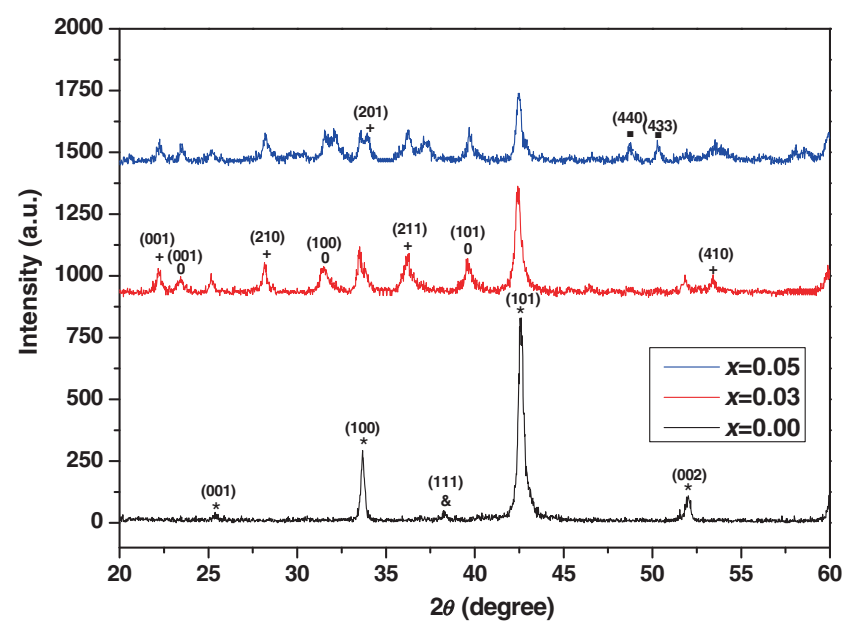

Figure 1. X-ray diffraction patterns for undoped and Er-doped $\mathrm{MgB}_{2}$ samples. $\mathrm{MgB}_{2}$ peaks are indicated with * symbol. Impurity peaks are indicated with the following symbols: \& for $\mathrm{MgO}, \boldsymbol{\square}$ for $\mathrm{Er}_{2} \mathrm{O}_{3}$, + for $\mathrm{ErB}_{4}$ and 0 for $\mathrm{ErB}_{2}$, respectively. 
Table 1. Data for Er-doped $\mathrm{MgB}_{2}$ samples.

\begin{tabular}{lcccccccccc}
\hline Samples & $\begin{array}{c}\text { Lattice } \\
\text { parameter, } a(\AA)\end{array}$ & $\begin{array}{c}\text { Lattice } \\
\text { parameter, } c(\AA)\end{array}$ & $\begin{array}{c}T_{\mathrm{c}}^{\text {onset }}(\mathrm{K}) \\
(\mathrm{K})\end{array}$ & $\begin{array}{c}\Delta T_{\mathrm{c}} \\
J_{\mathrm{c}}(4.2 \mathrm{~K}, 3 \mathrm{~T}) \\
\left.(\mathrm{A} \mathrm{cm})^{-2}\right)\end{array}$ & $\begin{array}{c}J_{\mathrm{c}}(15 \mathrm{~K}, 3 \mathrm{~T}) \\
\left(\mathrm{A} \mathrm{cm}^{-2}\right)\end{array}$ & $\begin{array}{c}\rho(300 \mathrm{~K}) \\
(\mu \Omega \mathrm{cm})\end{array}$ & $\begin{array}{l}\rho(40 \mathrm{~K}) \\
(\mu \Omega \mathrm{cm})\end{array}$ & $\begin{array}{l}\mathrm{RRR} \\
A_{\mathrm{F}}\end{array}$ \\
\hline$x=0.00$ & 3.073 & 3.511 & 38.63 & 1.97 & $2.2 \times 10^{3}$ & $0.6 \times 10^{3}$ & 1158.08 & 613.86 & 1.89 & 0.013 \\
$x=0.03$ & 3.074 & 3.523 & 38.49 & 0.67 & $1.1 \times 10^{5}$ & $6.2 \times 10^{4}$ & 249.07 & 103.09 & 2.42 & 0.050 \\
$x=0.05$ & 3.082 & 3.533 & 38.57 & 0.89 & $8.3 \times 10^{4}$ & $3.9 \times 10^{4}$ & 609.85 & 259.86 & 2.35 & 0.021 \\
\hline
\end{tabular}

sample (see figure 2a). The porous structure decreases and the homogeneity increases with Er doping (see figure $2 \mathrm{~b}$ and c). Also, it can be seen that the $\mathrm{MgB}_{2}$ grains are smaller for the doped samples compared to the undoped one. It leads to an increase in the surface area of grain boundaries, which act as pinning centres in $\mathrm{MgB}_{2}$.

Figure 3 shows the normal state resistivity vs. temperature measured at different applied magnetic fields up to $10 \mathrm{~T}$ for all samples over a temperature range of 10-300 K. The inset shows the resistivity $v s$. temperature curves at the temperatures between 10 and $45 \mathrm{~K}$. It can be seen that the scattering in the undoped porous $\mathrm{MgB}_{2}$ sample decreases with the increasing Er content. The resistivity at 40 and $300 \mathrm{~K}$ decreases from extraordinarily high value of 613.86 and $1158.08 \mu \Omega \mathrm{cm}$, respectively, for the porous $\mathrm{MgB}_{2}$, to 103.09 and $249.07 \mu \Omega \mathrm{cm}$, respectively, for the $3 \mathrm{wt} \% \mathrm{Er}-$ doped $\mathrm{MgB}_{2}$ sample. Such high resistivity of the undoped sample can be a result of the presence of pores between the superconducting grains. As known, dense $\mathrm{MgB}_{2}$-based materials have a significantly lower resistivity. ${ }^{6}$ Hence, the relatively lower resistivity may be related to higher sample density and better inter-granular connectivity. In a polycrystalline bulk sample produced by hot press method at $700^{\circ} \mathrm{C}$, the resistivity values are 2050 and $1560 \mu \Omega \mathrm{cm}$ at 300 and $50 \mathrm{~K}$, respectively, as reported by Rogado et $\mathrm{al}^{28}$ (the resistivity values have been taken from the small figures in Rogado et $\left.a l^{28}\right)$. Also, Sharma et $a l^{29}$ have reported the $T_{\mathrm{c}}$ value of the $\mathrm{MgB}_{2}$ sample, which was deliberately made with $\mathrm{Mg}$ deficiency, is to be around $39 \mathrm{~K}$ even as the resistivity at 300 $\mathrm{K}$ of the sample is above $100 \mathrm{~m} \Omega \mathrm{cm}$. From these results, it can be said that the resistivity values of the doped and undoped samples are compatible with literature. The resistivity of $5 \mathrm{wt} \%$ Er-doped sample is higher than the resistivity of $3 \mathrm{wt} \%$ Er-doped sample. It is probably the result of the excess impurity phases. It can be seen from figure 3 that all samples show a sharp superconducting transition at around 38-39 $\mathrm{K}$ in zero field regime. However, the transition range becomes narrower for the doped samples. The $T_{\mathrm{c}}^{\text {onset }}$ and the $\Delta T_{\mathrm{c}}$ values determined from figure 3 have been obtained to be $38.63,38.49,38.57 \mathrm{~K}$ and $1.97,0.67,0.89 \mathrm{~K}$ for 0,3 and $5 \mathrm{wt} \%$ Er-doped samples, respectively (see table 1 ). The $T_{\mathrm{c}}^{\text {onset }}$ value of the undoped sample is acceptable, because in some high resistivity $\mathrm{MgB}_{2}$ samples, it has been reported that $T_{\mathrm{c}}$ is surprisingly over $35 \mathrm{~K} .{ }^{30}$ Also, the minute suppression in $T_{\mathrm{c}}^{\text {onset }}$ values supports the partial substitution of Er into $\mathrm{MgB}_{2}$ lattice. The narrower transition to superconductivity for the doped samples implies the improvement in homogeneity and
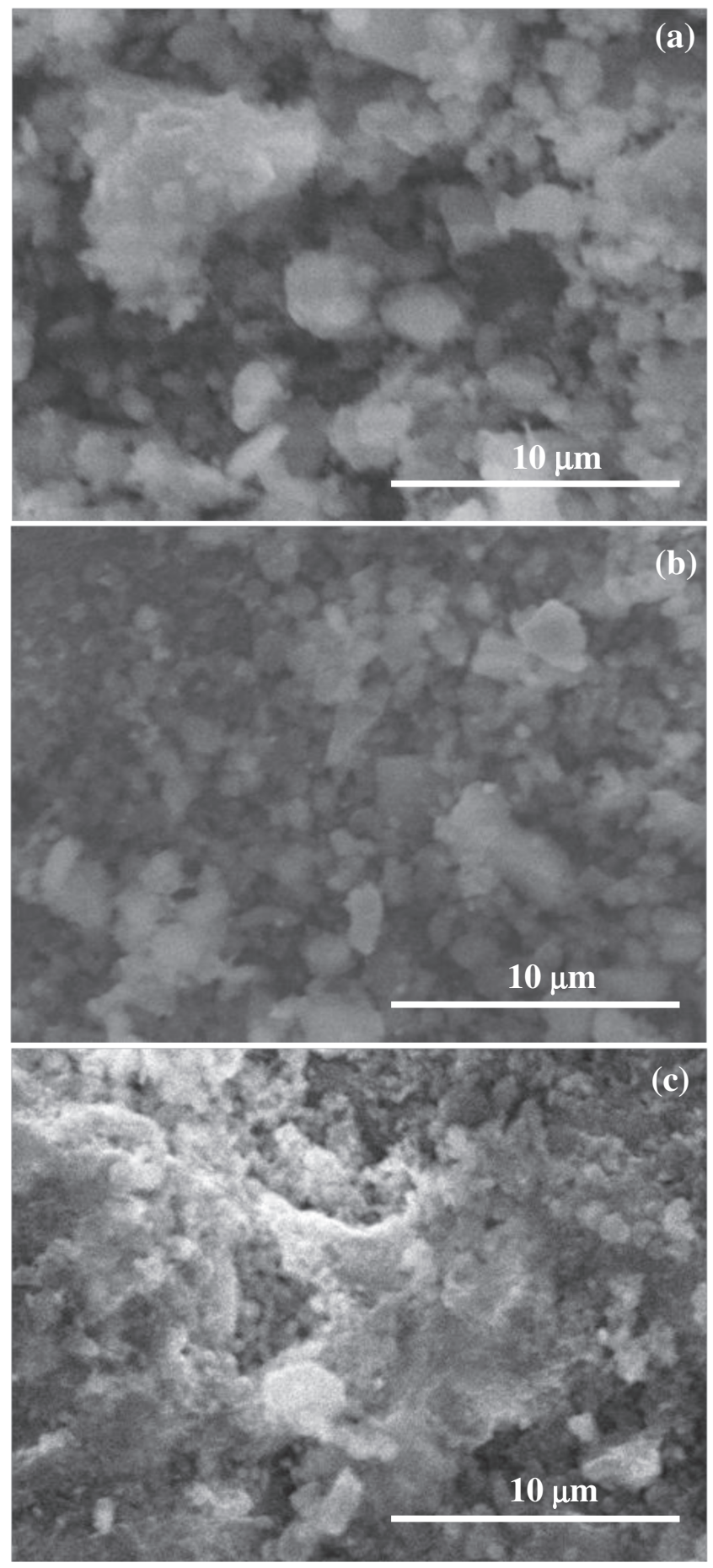

Figure 2. SEM images of (a) $x=0.00$, (b) $x=0.03$ and (c) $x=0.05$ Er-doped $\mathrm{MgB}_{2}$ samples. 
grain connectivity. The transition temperatures of all samples decrease as usual with the increasing applied field, as seen from figure 3 .

The residual resistivity ratios RRR related with the intragrain effect of grain boundary junctions have been calculated from the resistivity measurements $(\mathrm{RRR}=\rho(300 \mathrm{~K}) / \rho$ $(40 \mathrm{~K}))$. Also, the active area fractions $\left(A_{\mathrm{F}}\right)$ related with intergrain effect, have been evaluated by using the formula $A_{\mathrm{F}}=\Delta \rho_{\text {ideal }} /[\rho(300 \mathrm{~K})-\rho(40 \mathrm{~K})]$. Here, $\Delta \rho_{\text {ideal }}$ is the ideal change in resistivity from 300 to $40 \mathrm{~K}$ for a fully connected sample, for which the value of $7.3 \mu \Omega \mathrm{cm}$ is typically used. ${ }^{30,31}$ The residual resistivity ratios and the active area fractions for the undoped, 3 and $5 \mathrm{wt} \%$ Er-doped samples are $1.89,2.42,2.35$ and $0.013,0.050,0.021$, respectively, as shown in table 1. It is observed that the RRR and $A_{\mathrm{F}}$ values are higher for the doped samples than that of the undoped one. It is well known that the relatively high values of RRR indicate good quality of the samples. The fact that RRR shows an increasing trend is possibly due to the decrement of the grain sizes and the substitution of $\mathrm{Er}$ in $\mathrm{MgB}_{2}$. The increase in $A_{\mathrm{F}}$ of the doped samples signals decreasing porosity, improving grain boundary and a better connectivity.

The temperature dependence of $B_{\mathrm{c} 2}$ and $B_{\text {irr }}$ for all samples is shown in figure 4 . The $B_{\mathrm{irr}}$ and $B_{\mathrm{c} 2}$ at various temperatures for the samples have been estimated from the resistive transition curves using the criteria of 0.1 and 0.9 of $\rho\left(B, T_{\mathrm{c}}\right)$, respectively. ${ }^{25}$ It is observed that the $B_{\mathrm{c} 2}$ and $B_{\text {irr }}$ values are significantly enhanced for the doped samples. The best $B_{\mathrm{c} 2}$ and $B_{\text {irr }}$ increments have been obtained for the $3 \mathrm{wt} \% \mathrm{Er}$ doped sample, because the slope of $\mathrm{d} B_{\mathrm{c} 2} / \mathrm{d}\left(T / T_{\mathrm{c}}\right)$ for the $3 \mathrm{wt} \%$ Er-doped sample is larger compared to those for both the undoped and the $5 \mathrm{wt} \%$ Er-doped samples. For the best sample $(x=0.03)$ the $B_{\text {irr }}$ value at $20 \mathrm{~K}$ reaches $9.7 \mathrm{~T}$. It can be expected that the improvement in $B_{\text {irr }}$ is because of the flux pinning induced by grain boundaries, which increase in the doped samples due to the suppression of grain growth. In addition, the reason of the increment in $B_{\mathrm{c} 2}$ is probably the partial substitution of Er into $\mathrm{MgB}_{2}$ crystal lattice.

The $J_{\mathrm{c}}$ characteristic of all samples have been estimated from the high field magnetization measurements $M(H)$, by invoking Bean's critical state model. ${ }^{26}$ In this manner, the $J_{\mathrm{c}}(B)$ results are plotted in figure 5 at the temperatures between 4.2 and $35 \mathrm{~K}$. As seen from this figure, the $J_{\mathrm{c}}$ of the doped samples are higher than that of the undoped one for all fields and at all temperatures. The values of $J_{\mathrm{c}}$ at $4.2 \mathrm{~K}$ and under a field of $3 \mathrm{~T}$ have been calculated to be $2.2 \times 10^{3} \mathrm{~A} \mathrm{~cm}^{-2}$ for the undoped $\mathrm{MgB}_{2}$ sample, $1.1 \times 10^{5} \mathrm{~A} \mathrm{~cm}^{-2}$ for $3 \mathrm{wt} \%$ Er-doped sample and $8.3 \times 10^{4} \mathrm{~A} \mathrm{~cm}^{-2}$ for $5 \mathrm{wt} \%$ Er-doped sample. The values of $J_{\mathrm{c}}$ under a field of $3 \mathrm{~T}$ and at the temperatures of 4.2 and $15 \mathrm{~K}$ have been listed in table 1 . For the best sample $(x=0.03)$, at $15 \mathrm{~K}$ under a magnetic field of $4 \mathrm{~T}$, the $J_{\mathrm{c}}$ value reaches $2.4 \times 10^{4} \mathrm{~A} \mathrm{~cm}^{-2}$, which is higher than that of the undoped sample by an order of $10^{3}$ (see figure 5). The $J_{\mathrm{c}}$ values show clearly that the Er-doped samples exhibit better current-carrying capabilities under external magnetic fields. This is an indication of the enhancement in the flux

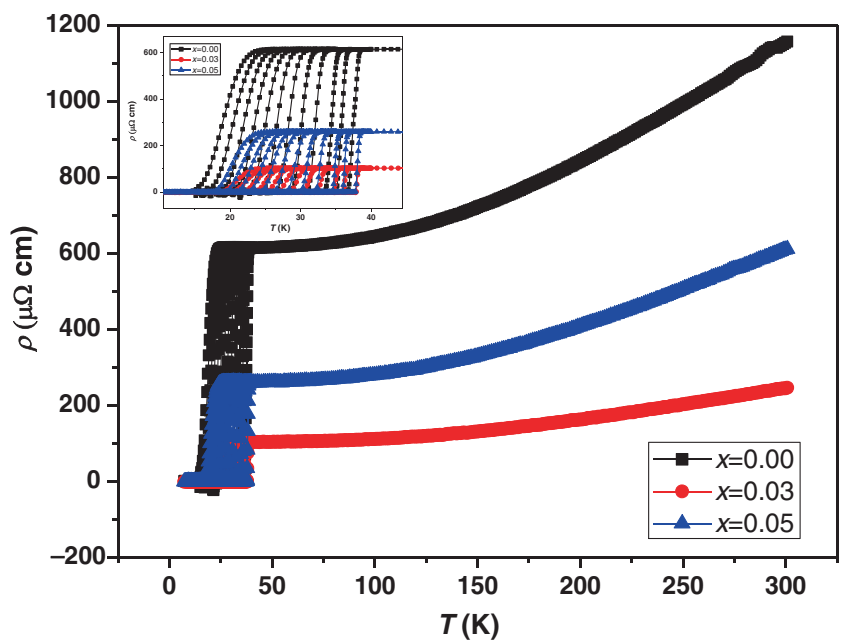

Figure 3. Superconducting transition zone resistivity $v s$. temperature from 10 to $300 \mathrm{~K}$ under different applied magnetic fields up to $10 \mathrm{~T}$ for undoped and Er-doped $\mathrm{MgB}_{2}$ samples. The inset shows the resistivity at the temperatures between 10 and $45 \mathrm{~K}$.

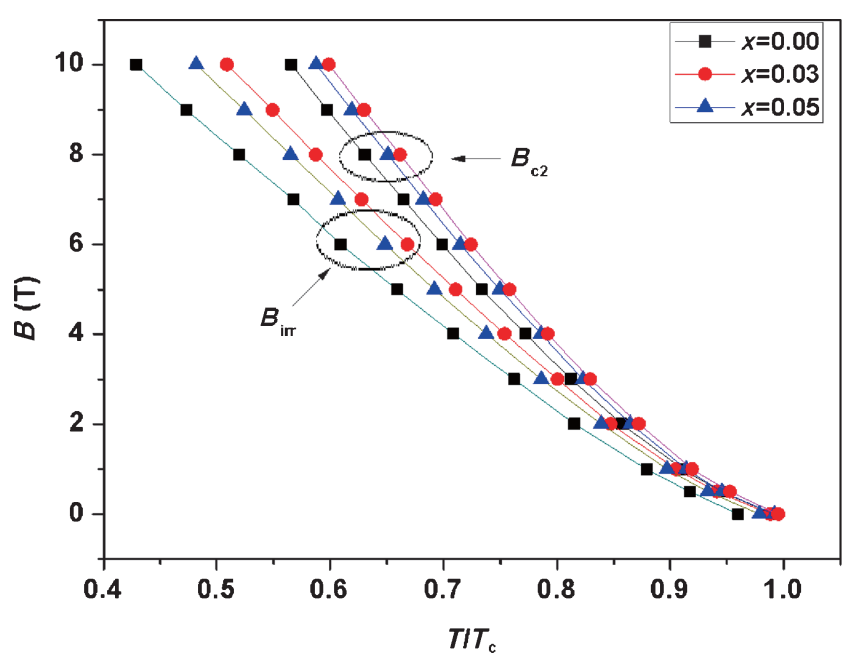

Figure 4. The upper critical field $B_{\mathrm{c} 2}$ and the irreversibility field $B_{\text {irr }}$ as functions of $T / T_{\mathrm{c}}$ for the samples synthesized with the nominal composition $\left(\mathrm{Mg}_{1-x} \mathrm{Er}_{x}\right) \mathrm{B}_{2}$.

pinning strength of the doped samples. The $J_{\mathrm{c}}$ variation with the increasing Er content are compatible with the resistivity results (see table 1), as reported by Rowell ${ }^{30}$ that an important implication of the high resistivity, if they are indeed due to limitations in the area of the sample that carries current, is that exactly the same limitations should apply to the sample area that carries supercurrent. It might be expected that the improvement in $J_{\mathrm{c}}$ of the doped samples is related with the depression on the growth rate of $\mathrm{MgB}_{2}$ grains by Er doping and so, the increment in the surface area of the grain boundaries, which act as pinning centres in $\mathrm{MgB}_{2}$. Also, the impurity phases can act as effective pinning centres in the doped samples. 


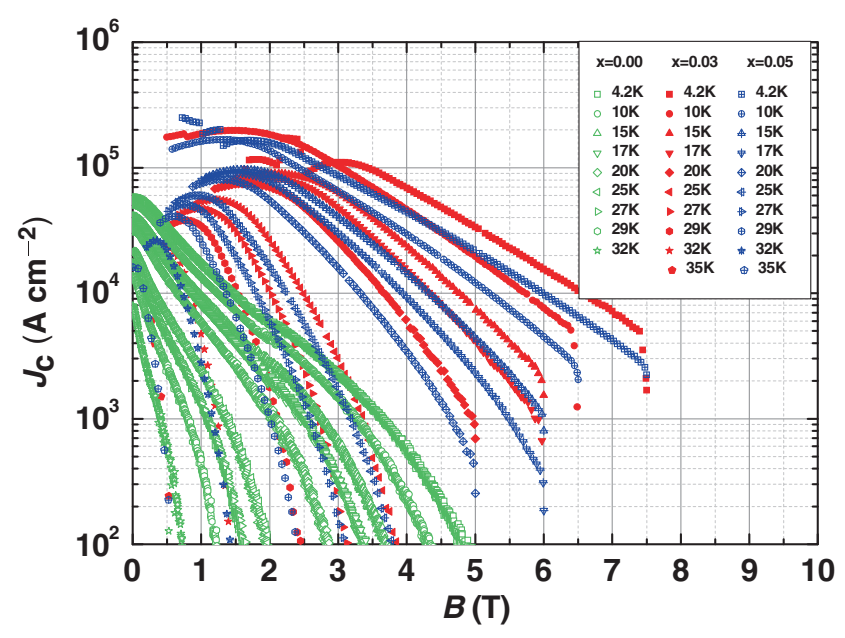

Figure 5. Magnetic field $B$ dependence of the critical current density $J_{\mathrm{c}}$ for the undoped and Er-doped $\mathrm{MgB}_{2}$ samples at the temperatures between 4.2 and $35 \mathrm{~K}$.

\section{Conclusions}

In this study, the effects of Er doping on the superconducting properties of porous $\mathrm{MgB}_{2}$ have been systematically studied. The $B_{\mathrm{c} 2}, B_{\text {irr }}$ and $J_{\mathrm{c}}$ values are significantly enhanced for the doped samples, due to the degradation of porosity, the reduction in grain size and thus, the enhancement on grain connectivity and grain boundary pinning. Substantially enhanced $B_{\mathrm{c} 2}, B_{\text {irr }}$ and $J_{\mathrm{c}}$ values have been observed in the $3 \mathrm{wt} \%$ Er-doped sample. It can be concluded from this experimental study that the RE element $\mathrm{Er}$ is an excellent candidate as a doping source for improving the superconducting properties of $\mathrm{MgB}_{2}$, without significantly affecting the $T_{\mathrm{c}}$ of the material.

\section{References}

1. Nagamatsu J, Nakagawa N, Muranaka T, Zenitani Y and Akimitsu J 2001 Nature $\mathbf{4 1 0} 63$

2. Kim H J, Kang W N, Choi E M, Kim M S, Kim K H P and Lee S I 2001 Phys. Rev. Lett. 87087002

3. Lyard L, Szabó P, Klein T, Marcus J, Marcenat C, Kim K H, Kang B W, Lee H S and Lee S I 2004 Phys. Rev. Lett. 92 057001

4. Larbalestier D C et al 2001 Nature $\mathbf{4 1 0} 186$

5. Liu C F, Yan G, Du S J, Xi W, Feng Y, Zhang P X, Wu X Z and Zhou L 2003 Physica C 386603

6. Putti M, Braccini V, Galleani E, Napoli F, Pallecchi I, Siri A S, Manfrinetti P and Palenzona A 2003 Supercond. Sci. Technol. 16188
7. Rowell J M, Xu S Y, Zeng X H, Pogrebnyakov A V, Li Q, Xi X X, Redwing J M, Tian W and Pan X 2003 Appl. Phys. Lett. 83102

8. Li H L, Ruan K O, Li S Y, Yu Y, Wang C Y and Cao L Z 2003 Physica C 386560

9. Yao C, Zhang X, Wang D, Gao Z, Wang L, Qi Y, Wang C, Ma Y, Awaji S and Watanabe K 2011 Supercond. Sci. Technol. 24 055016

10. Bugoslavsky Y, Cohen L F, Perkins G K, Polichetti M, Tate T J, Gwilliam R and Caplin A D 2001 Nature 411561

11. Dou S X, Soltanian S, Horvat J, Wang X L, Zhou S H, Ionescu M, Liu H K, Munroe P and Tomsic M 2002 Appl. Phys. Lett. 813419

12. Zhao Y, Feng $\mathrm{Y}$, Cheng $\mathrm{C} \mathrm{H}$, Zhou L, Wu Y, Machi T, Fudamoto Y, Koshizuka N and Murakami M 2001 Appl. Phys. Lett. 791154

13. Slusky J S et al 2001 Nature (London) 410343

14. Felner I 2001 Physica C 35311

15. Feng Y, Zhao Y, Sun Y P, Liu F C, Fu B Q, Zhou L, Cheng C H, Koshizuka N and Murakami M 2001 Appl. Phys. Lett. 793893

16. Kuhberger M and Gritzner G 2002 Physica C 37039

17. Kitaguchi H and Kumakura H 2005 Supercond. Sci. Technol. $18 \mathrm{~S} 284$

18. Cheng $\mathrm{C} \mathrm{H}$, Zhao Y, Zhu X T, Nowotny J, Sorrell C C, Finlayson T and Zhang H 2003 Physica C 386588

19. Wang J, Bugoslavsky Y, Berenov A, Cowey L, Caplin A D, Cohen L F, Cooley L D, Song X and Larbalestier D C 2002 Appl. Phys. Lett. 812026

20. Chen S K, Wei M and MacManus-Driscoll J L 2006 Appl. Phys. Lett. 88192512

21. Pan X F, Shen T M, Li G, Cheng C H and Zhao Y 2007 Phys. Status Solidi (a) 2041555

22. Katsura Y, Shimoyama J, Yamamoto A, Horii S and Kishio K 2007 Physica C 463-465 225

23. Ojha N, Varma G D, Singh H K and Awana V P S 2009 J. Appl. Phys. 105 07E315

24. Cheng C and Zhao Y 2006 Appl. Phys. Lett. 89252501

25. Hossain M S A, Kim J H, Xu X, Wang X L, Rindfleisch M, Tomic M, Sumption M D, Collings E W and Dou S X 2007 Supercond. Sci. Technol. 20 L51

26. Bean C P 1962 Phys. Rev. Lett. 8250

27. Grinenko V, Krasnoperov E P, Stoliarov V A, Bush A A and Mikhajlov B P 2006 Solid State Commun. 138461

28. Rogado N, Hayward M A, Regan K A, Wang Y, Ong N P, Zandbergen H W, Rowell J M and Cava R J 2002 J. Appl. Phys. 91274

29. Sharma P A, Hur N, Horibe Y, Chen C H, Kim B G, Guha S, Cieplak M Z and Cheong S W 2002 Phys. Rev. Lett. 89167003

30. Rowell J M 2003 Supercond. Sci. Technol. 16 R17

31. Jiang J, Senkowicz B J, Larbalestier D C and Hellstrom E E 2006 Supercond. Sci. Technol. 19 L33 